\title{
MULTI-STAGE RESIDUAL HIDING FOR IMAGE-INTO-AUDIO STEGANOGRAPHY
}

\author{
Wenxue Cui ${ }^{\star} \quad$ Shaohui Liu ${ }^{\star} \quad$ Feng Jiang ${ }^{\star} \quad$ Yongliang Liu ${ }^{\dagger} \quad$ Debin Zhao \\ * Harbin Institute of Technology, Harbin, China $\quad$ * Peng Cheng Laboratory, Shenzhen China \\ $\dagger$ Alibaba Group, Hangzhou, China
}

\begin{abstract}
The widespread application of audio communication technologies has speeded up audio data flowing across the Internet, which made it a popular carrier for covert communication. In this paper, we present a cross-modal steganography method for hiding image content into audio carriers while preserving the perceptual fidelity of the cover audio. In our framework, two multi-stage networks are designed: the first network encodes the decreasing multilevel residual errors inside different audio subsequences with the corresponding stage sub-networks, while the second network decodes the residual errors from the modified carrier with the corresponding stage sub-networks to produce the final revealed results. The multi-stage design of proposed framework not only make the controlling of payload capacity more flexible, but also make hiding easier because of the gradual sparse characteristic of residual errors. Qualitative experiments suggest that modifications to the carrier are unnoticeable by human listeners and that the decoded images are highly intelligible.
\end{abstract}

Index Terms - Audio steganography, residual hiding, multi-stage network, convolutional neural networks

\section{INTRODUCTION}

Sometimes a file carries more information than it perceptively conveys. To a casual observer, a file may appear normal, but knowledgeable recipients can extract more information from it. Recently, to protect confidential data, steganography has been researched extensively, which is a technique of concealing secret messages in digital carriers to facilitate covert communication through exploiting the redundancy of human perceptions. The secrecy characteristics of steganography are attractive for diverse applications such as copyright certification [1] and covert communication [2].

Faithful hiding heavily depends on its embedding method. To achieve perfect hiding performance, a wide variety of steganography setting and methods have been proposed. For instance, Least Significant Bit (LSB) substitution methods [3, 4, 5] have been extremely popular for steganography due to their simplicity. Then some advanced methods emerged such as HUGO [6], WOW [7] and S-UNIWARD [8], which embedded the messages in complex textures by min- imizing a well-crafted distortion function and achieved superior performance. However, the aforementioned methods usually use domain knowledge to identify features for hiding secret message into cover carriers, which usually leads to small payload capacities and apparent distortion altering.

In recent years, deep neural networks (DNN) have recently been applied to steganography, with a strong focus on images. Instead of specifying domain knowledge explicitly, DNN-based methods have been explored to learn the signal characteristics implicitly. The earliest application of deep learning to steganography was based on Generative Adversarial Networks (GAN) [9, 10, 11, 12, 13]. The introduction of the adversarial training not only resists more kinds of attacks but also achieves better visual performance. In order to enhance the payload capacities of steganography, several steganography algorithms [1, 14, 15] that embed an image into another image are proposed. Furthermore, with the popularization of diverse audio applications, researchers begin to pay more attention to speech steganography. In [16], two neural networks are optimized jointly to embed message into the cover audio and extract message from the modified carrier. In [17], a GAN-based audio-to-audio framework is proposed, in which an encoder network and a decoder network is designed for information embedding and extraction in the frequency domain by using short-time fourier transform.

Compared with the hand-crafted embedding methods, the aforementioned deep network-based steganography methods have achieved superior performance. However, these frameworks still exist the following weaknesses: (1) Most of these methods seem to be powerless for the steganography between different data modalities. (2) Hiding the secret message directly is difficult because of its diversity of knowledge and therefore usually leads to noticeable artifacts.

To overcome the shortcomings of the aforementioned methods, we propose a Deep neural network based Image-ToAudio Steganography (DITAS) framework as shown in Fig.1, in which two multi-stage networks are designed: hiding network and revealing network. Specifically, the hiding network encodes the decreasing residual errors inside different audio subsequences with the corresponding stage sub-networks, and the revealing network decodes the residual errors from the modified carrier with the corresponding stage sub-networks to produce the final revealed results. The proposed framework 


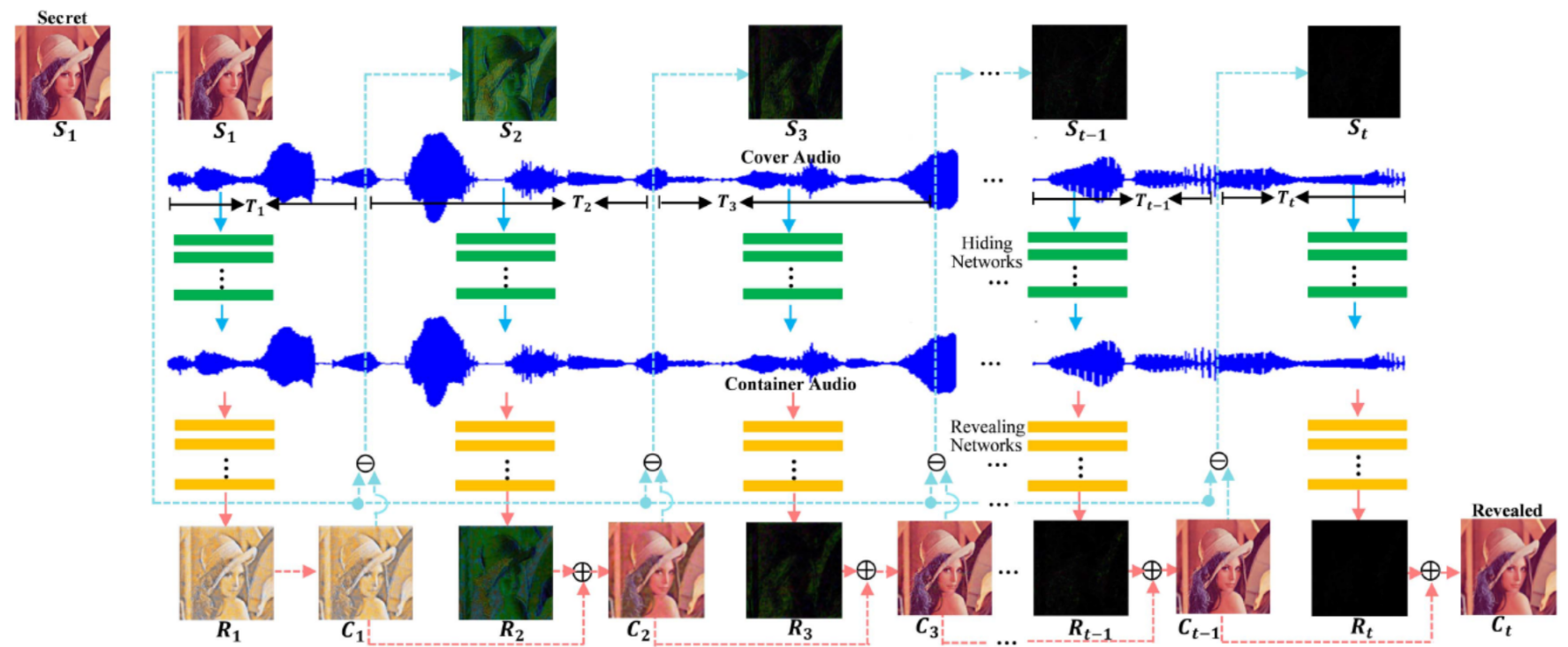

Fig. 1. Diagram of our proposed steganography framework.

hides the secret message in an progressive fashion, which makes hiding process more easier. Experimental results on benchmark datasets demonstrate that the proposed method is capable of achieving superior performance against other algorithms.

The main contributions are summarized as follows:

1) We propose a novel image-to-audio steganography framework based on deep learning, which achieves superior hiding capabilities against other methods.

2) By hiding the residual errors of multiple levels, the proposed method not only can control the payload capacity more flexibly, but also make the hiding process more easier.

3) Our framework embed the residual errors into different audio subsequences, which implies that even if part of the carrier is lost, the secret image can be restored to some extent.

\section{PROPOSED METHOD}

\subsection{Hiding Network}

Given a secret image $S_{0}$ with size $w \times h$ and a cover audio $A_{l}$, where $w, h$ are the width and height of secret image and $l$ indicates the dimension of cover audio. We embed multilevel residual errors of secret image into cover audio progressively by using a multi-stage network. Specifically, $t$ non-overlapping audio subsequences are first selected from the cover audio sequence, which expressed mathematically as $\left\{T_{1}, T_{2}, \ldots, T_{t}\right\}$ and the dimension of each subsequence is $w * h$. The proposed framework consists of $t$ stages to embed residual errors of the secret image into these $t$ subsequences correspondingly. More concretely, for the $i$-th stage, we hide the residual error between the original secret image and the revealed results from the previous $i-1$ stages revealing subnetworks. The hiding process can be expressed as

$$
\tilde{T}_{i}=\mathcal{H}\left(S_{i}, T_{i} ; \theta_{H_{i}}\right)
$$

where $\mathcal{H}$ indicates the operation of hiding network and $\theta_{H_{i}}$ is the parameter of $i$-th stage hiding sub-network. $S_{i}$ is the $i$-th level residual error of secret image to be hidden and $\tilde{T}_{i}$ is the hidden result (Container) that preserves the perceptual fidelity of the cover audio $T_{i}$. The residual error $S_{i}$ can be expressed as

$$
S_{i}=S_{0}-C_{i-1}, \quad C_{i-1}=\sum_{j=0}^{i-1} R_{j}
$$

where $S_{0}$ is the original secret image, $R_{i}(i>0)$ indicates the revealed result of $i$-th stage revealing sub-network, which will be demonstrated in the next subsection and $R_{0}$ is a zero tensor with the same size of $S_{0} . C_{i}$ indicates the total sum of revealed results of previous $i$ stages revealing sub-networks.

\subsection{Revealing Network}

After hiding stage, the hidden audio subsequence $\tilde{T}_{i}$ is produced. Subsequently, the goal of revealing network is to extract the secret image from the hidden audio sequence in the revealing stage. Specifically, given the hidden audio subsequence $\tilde{T}_{i}$, the revealing process can be expressed as

$$
R_{i}=\mathcal{R}\left(\tilde{T}_{i} ; \theta_{R_{i}}\right)
$$

where $\mathcal{R}$ indicates the operation of revealing network and $\theta_{R_{i}}$ is the parameter of its $i$-th stage revealing sub-network. $R_{i}$ is the revealed residual result from $\tilde{T}_{i}$. After get the residuals $R_{i}$, we add them together to produce the final revealed

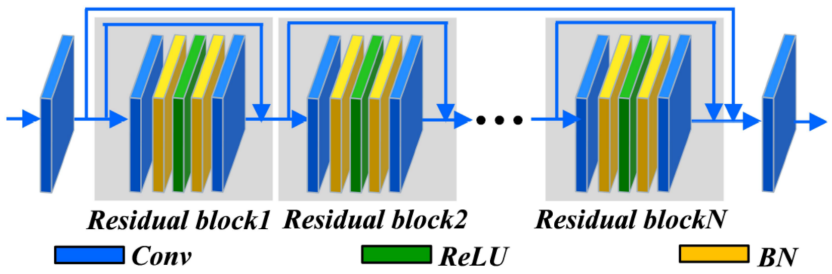

Fig. 2. The architecture of proposed residual block. 
Table 1. The performances for different deep learning-based steganography algorithms. Bold indicates the best performance.

\begin{tabular}{c||c||c|c|c||c||c|c|c}
\hline \hline \multicolumn{1}{c||}{ Datasets } & \multicolumn{4}{c||}{ Natural image $\rightarrow$ TIMIT } & \multicolumn{4}{c}{ Face image $\rightarrow$ TIMIT } \\
\hline \multirow{2}{*}{ Method } & Container & \multicolumn{3}{|c||}{ Revealed } & Container & \multicolumn{3}{c}{ Revealed } \\
\cline { 2 - 9 } & MSE & PSNR & SSIM & MS-SSIM & MSE & PSNR & SSIM & MS-SSIM \\
\hline \hline Deep-Steg [1] & $2.7669 \mathrm{E}-3$ & 31.40 & 0.8624 & 0.9576 & $2.2258 \mathrm{E}-3$ & 31.44 & 0.8196 & 0.9380 \\
\hline Kreuk'Model [16] & $1.3495 \mathrm{E}-3$ & 33.68 & 0.8893 & 0.9671 & $\mathbf{9 . 8 8 7 2 E}-4$ & 33.59 & 0.8420 & 0.9574 \\
\hline DITAS-S & $1.2144 \mathrm{E}-3$ & 27.84 & 0.8691 & 0.9685 & $1.1033 \mathrm{E}-3$ & 28.05 & 0.7682 & 0.9340 \\
\hline DITAS-M & $1.9249 \mathrm{E}-3$ & 37.09 & 0.9444 & 0.9925 & $2.1313 \mathrm{E}-3$ & 36.12 & 0.8898 & 0.9807 \\
\hline DITAS-M-E & $\mathbf{9 . 3 8 6 4 E - 4}$ & 37.84 & 0.9482 & 0.9934 & $9.9386 \mathrm{E}-4$ & 36.52 & 0.8906 & 0.9847 \\
\hline DITAS-M-D & $1.6904 \mathrm{E}-3$ & $\mathbf{3 8 . 4 2}$ & 0.9581 & 0.9935 & $1.7932 \mathrm{E}-3$ & 37.65 & 0.9036 & 0.9883 \\
\hline DITAS-M-ED & $9.8471 \mathrm{E}-4$ & 38.39 & $\mathbf{0 . 9 5 9 7}$ & $\mathbf{0 . 9 9 4 4}$ & $1.0582 \mathrm{E}-3$ & $\mathbf{3 7 . 6 9}$ & $\mathbf{0 . 9 0 5 4}$ & $\mathbf{0 . 9 9 0 0}$ \\
\hline \hline
\end{tabular}

result, namely $C_{i}=\sum_{j=0}^{i} R_{j}$. It is worth emphasizing that the proposed framework embeds multi-level residuals in different audio subsequences and therefore the revealed result is extracted progressively from the container sequence. In other words, the subsequences are independent between each other and even if some subsequences are lost, the secret image can also be revealed to some extent, which improves the robustness of the proposed method.

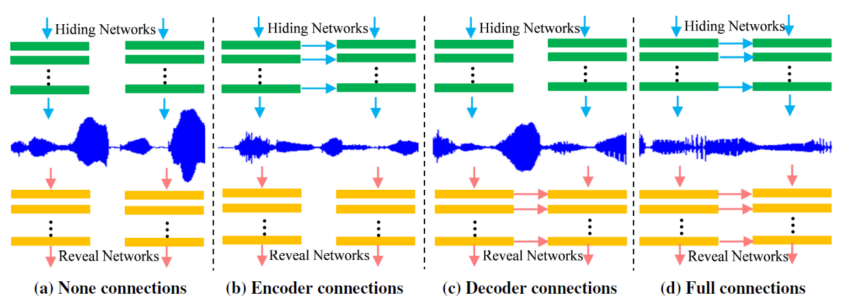

Fig. 3. The structural details of four experimental variants.

\subsection{Loss Function}

In the proposed framework, we have two main missions: one is secret image hiding, the other is secret image extraction. Therefore, there are two loss items to constraint the container and the extracted entities, respectively. Besides, the proposed framework consists of $t$ stages and therefore the complete loss function can be expressed as:

$$
\mathcal{L}\left(\theta_{H_{i}}, \theta_{R_{i}}\right)=\sum_{i=1}^{t} \mathcal{L}_{H_{i}}\left(\theta_{H_{i}}\right)+\lambda_{i} \mathcal{L}_{R_{i}}\left(\theta_{R_{i}}\right)
$$

where $\mathcal{L}_{H_{i}}$ is hiding loss of $i$-th stage hiding sub-network for information concealing and $\mathcal{L}_{R_{i}}$ is revealing loss of $i$-th stage revealing sub-network for information extraction. $\theta_{H_{i}}$ and $\theta_{R_{i}}$ are the parameters of them. $\lambda_{i}$ is the regularization parameter to control the tradeoff between them. Specifically, the hiding loss is defined as

$$
\mathcal{L}_{H_{i}}\left(\theta_{H_{i}}\right)=\frac{1}{N} \sum_{i=1}^{N}\left\|\mathcal{H}\left(S_{i}, T_{i} ; \theta_{H_{i}}\right)-T_{i}\right\|_{2}^{2}
$$

On the other hand, to ensure the precision of extracted information, another extraction loss is defined

$$
\mathcal{L}_{R_{i}}\left(\theta_{R_{i}}\right)=\frac{1}{N} \sum_{i=1}^{N}\left\|\mathcal{R}\left(\tilde{T}_{i} ; \theta_{R_{i}}\right)-S_{i}\right\|_{2}^{2}
$$

where $\tilde{T}_{i}=\mathcal{H}\left(S_{i}, T_{i} ; \theta_{H_{i}}\right)$ indicates the container audio sequences.

\section{EXPERIMENTAL RESULTS AND ANALYSIS}

\subsection{Implementation and training details}

In order to embed the secret image into the cover audio by convolutional operations, preprocessing is needed for the onedimensional audio subsequence $T_{i}$. Specifically, two methods of audio data pre-processing are conducted: 1) Raw audio data is reshaped directly to a new tensor. 2) Short-Time Fourier Transform (STFT) is applied to transform audio from the audio domain to the frequency domain.

After the pre-processing, a tensor with size of $w \times h$ is produced for $T_{i}$, which can be concatenated with the secret entity $S_{i}$ together conveniently as the input of $i$-th stage hiding subnetwork. In the proposed hiding and revealing networks, the proposed residual block is utilized as shown in Fig.2. Specifically, for each stage hiding sub-network, each convolutional layer consists of 64 kernels with size of $3 \times 3$ except for the last layer, in which single kernel is included to ensure dimensional consistency between the output and the cover audio tensor. Similarly, for the revealing network, the last convolutional layer has 3 kernels to extract the final revealed residuals and 64 kernels are included for the rest layers. We train our model with the python toolbox Pytorch [18] on a Titan X GPU. Adaptive moment estimation (Adam) [19] is utilized to optimize all network parameters. Furthermore, we set $\lambda_{i}=$
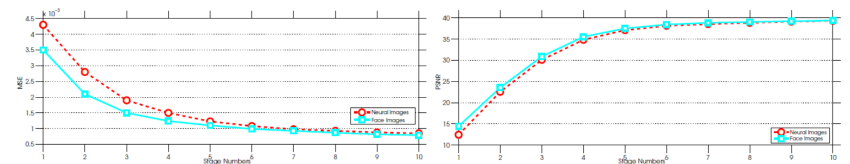

Fig. 4. The relationship between the number of stages and results based on audio dataset TIMIT. ( Left is the MSE of container audio and right is PSNR of revealed image). 


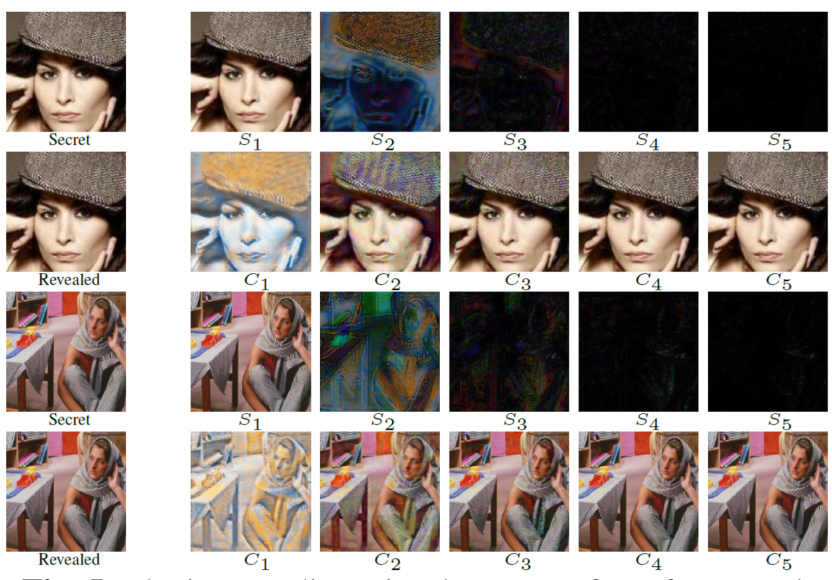

Fig. 5. The intermediate visual outputs of our framework.

0.8 . In order to choose the optimal number of stages $(t)$, we train a model with 10 stages and Fig. 4 shows the relationship between the performance and the stage numbers, from which we can see that with the increase of stages, the performance growth is gradually slow down and we set $t=5$ in our model. It is worth emphasizing that the proposed framework can be trained in an end-to-end fashion.

For training, we use the training set of dataset VOC2012 [20] as the secret image and use the LJ Speech dataset [21] as the cover audio. For testing, we choose 2 kinds of images as the secret image: natural image (including Set14 [22], LIVE1 [23], Val of VOC2012 [20] and Val of ImageNet [14]) and face image (including CelebA dataset [12]), which are widely used in the literatures. Besides, we use the TIMIT audio dataset [24] as the cover audio. For training details, the patch size is set as $64 \times 64$, which is cropped from training dataset randomly and we set batch size as 16 . The learning rate is initialized to $1 e-4$ for all layers and decreased by a factor of 3 for every 20 epochs. We train the entire network for 200 epochs.

\subsection{Comparison with other methods}

Following the deep steganography structure proposed by Baluja et al. [1], we can build a network model for hiding an image in audio. Specifically, given a cover audio, $t$ nonoverlapping sub-sequences with size of $w * h$ are first selected. For each subsequence, we reshape it as $w \times h$ and concatenate them together, after which we get a tensor with size $t \times w \times h$. Then, we embed the secret image into this tensor. Besides, refer to the previous work [16], we can also obtain the audio tensor by using STFT, and then embed the secret image into the frequency domain of the cover audio.

Considering the proposed framework, we try two preprocessing methods mentioned in subsection 3.1 for the cover audio transformation. We find that steganography in frequency domain is slightly better than that in raw audio data domain. While the execution of STFT will increase the computational complexity. Therefore, we reshape the raw audio data into tensor directly in this work. In the proposed framework, we train each stage subnetwork without any information transfer between them (shown in Fig.3(a)) and name it as "DITASM". Besides, to verify the efficiency of information transfer between stages, four experimental variants are designed: 1) We build the connections between successive stages of hiding subnetworks to propagate information (shown in Fig.3(b)) and we name it as "DITAS-M-E". 2) The connections between successive stages of revealing subnetwork are builded (shown in Fig.3(c)) and we name it as "DITAS-M-D". 3) Full connections are set up between neighbouring stages (shown in Fig.3(d)) and we name it as "DITAS-M-ED". 4) Shared parameters are utilized for each stage and we name it as "DITAS-S".

For experimental verification, we use Mean Square Error (MSE) to measure the distortion of hidden audio and utilize three evaluation metrics (PSNR, SSIM, MS-SSIM) to measure the quality of revealed image. Table.1 shows the objective experimental results, from which we can see that the proposed method can achieve superior performance against other methods and obtains more than $3 \mathrm{~dB}$ gains in terms of PSNR. Fig. 5 shows the intermediate outputs of our model, from which we can see that $S_{i}$ is more and more sparse and the vision of $C_{i}$ is better and better. Fig.6 shows the comparisons of subjective quality between the proposed method and others, from which we can see that the proposed method is capable of achieving better visual performance.
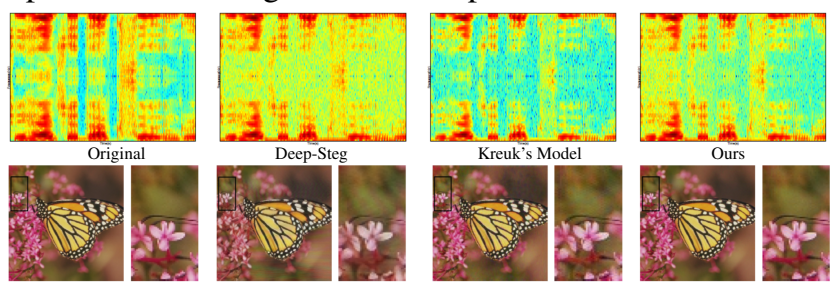

Fig. 6. The visual comparisons of different steganography methods. (Top is the visual comparisons of container in the frequency domain and bottom is the revealed perceptions.)

\section{CONCLUSION}

In this paper, we propose a novel cross-modal image-to-audio steganography framework based on deep learning. Instead of hiding the secret image directly, the proposed method embeds the residual errors of secret image into the cover audio progressively by a multi-stage fashion. In the hiding process of the proposed method, residual errors become more sparse with the increase of stages, which not only make the controlling of payload capacity more flexible, but also make hiding easier because of the sparsity characteristic of residual errors.

\section{ACKNOWLEGEMENT}

This work was supported by Alibaba Group through Alibaba Innovative Research (AIR) Program and partly funded by National Key Research and Development Program of China via grant 2018YFC0806802 and 2018YFC0832105. 


\section{REFERENCES}

[1] Shumeet Baluja, "Hiding images in plain sight: Deep steganography," Advances in Neural Information Processing Systems (NIPS), pp. 2069-2079, 2017.

[2] Eric Cole and Ronald D. Krutz, "Hiding in plain sight: Steganography and the art of covert communication," 2003.

[3] Ron G. Van Schyndel, Andrew Z. Tirkel, and Charles F. Osborne, "A digital watermark," in IEEE International Conference on Image Processing (ICIP), 1994.

[4] Raymond B. Wolfgang and Edward J. Delp, "A watermark for digital images," IEEE International Conference on Image Processing (ICIP), 1996.

[5] M. Asad, J. Gilani, and A. Khalid, "An enhanced least significant bit modification technique for audio steganography," in International Conference on Computer Networks and Information Technology, 2011, pp. 143-147.

[6] Tomáš Pevný, Tomáš Filler, and Patrick Bas, "Using high-dimensional image models to perform highly undetectable steganography," vol. 6387, pp. 161-177, 2010.

[7] Vojtěch Holub and Jessica Fridrich, "Designing steganographic distortion using directional filters," in IEEE Workshop on Information Forensic and Security, 2012.

[8] Vojtěch Holub, Jessica Fridrich, and Tomáš Denemark, "Universal distortion function for steganography in an arbitrary domain," Eurasip Journal on Information Security, pp. 1-13, 2014.

[9] Jiren Zhu, Russell Kaplan, Justin Johnson, and Li FeiFei, "Hidden: Hiding data with deep networks," Proceedings of the European Conference on Computer Vision (ECCV), pp. 657-672, 2018.

[10] Ian J Goodfellow, Jean Pouget-Abadie, Mehdi Mirza, $\mathrm{Xu}$ Bing, and Yoshua Bengio, "Generative adversarial nets," in International Conference on Neural Information Processing Systems (NIPS), 2014.

[11] Denis Volkhonskiy, Ivan Nazarov, and Evgeny Burnaev, "Steganographic generative adversarial networks," 2017.

[12] Haichao Shi, Jing Dong, Wei Wang, Yinlong Qian, and Xiaoyu Zhang, "Ssgan: Secure steganography based on generative adversarial networks," 2017.
[13] Jamie Hayes and George Danezis, "Generating steganographic images via adversarial training," in Advances in Neural Information Processing Systems 30, pp. 19541963. Curran Associates, Inc., 2017.

[14] Atique Ur Rehman, Rafia Rahim, M Shahroz Nadeem, and Sibt Ul Hussain, "End-to-end trained cnn encodedecoder networks for image steganography," 2017.

[15] Pin Wu, Yang Yang, and Xiaoqiang Li, "Imageinto-image steganography using deep convolutional network," in Advances in Multimedia Information Processing - PCM 2018 - 19th Pacific-Rim Conference on Multimedia, Hefei, China, September 21-22, 2018, Proceedings, Part II. 2018, vol. 11165 of Lecture Notes in Computer Science, pp. 792-802, Springer.

[16] Felix Kreuk, Yossi Adi, Bhiksha Raj, Rita Singh, and Joseph Keshet, "Hide and speak: Deep neural networks for speech steganography," arXiv preprint arXiv:1902.03083, 2019.

[17] Dengpan Ye, Shunzhi Jiang, and Jiaqin Huang, "Heard more than heard: An audio steganography method based on gan," arXiv preprint arXiv:1907.04986, 2019.

[18] Soumith Chintala Adam Paszke, Sam Gross and Gregory Chanan, "Pytorch: Tensors and dynamic neural networks in python with strong gpu acceleration," 2017.

[19] Diederik Kingma and Jimmy Ba, "Adam: A method for stochastic optimization," Computer Science, 2014.

[20] Shiqi Dong Ru Zhang and Jianyi Liu, "Invisible steganography via generative adversarial networks," 2018.

[21] Keith Ito, "The lj speech dataset. https://keithito.com/ljspeech-dataset/," 2017.

[22] Wuzhen Shi, Feng Jiang, Shengping Zhang, and Debin Zhao, "Deep networks for compressed image sensing," IEEE International Conference on Multimedia and Expo (ICME), pp. 877-882, 2017.

[23] Wenxue Cui, Feng Jiang, Xinwei Gao, Shengping Zhang, and Debin Zhao, "An efficient deep quantized compressed sensing coding framework of natural images," in 2018 ACM Multimedia Conference on Multimedia Conference, MM 2018, Seoul, Republic of Korea, October 22-26, 2018. 2018, pp. 1777-1785, ACM.

[24] Victor Zue, Stephanie Seneff, and James Glass, "Speech database development at mit: Timit and beyond," Speech Communication, vol. 9, no. 4, pp. 351-356, 1990. 